\title{
MODELOS DE AVALIAÇÃO PRÉ-CONSTRUÇÃO EM EMPREENDIMENTOS HABITACIONAIS DE INTERESSE SOCIAL: UMA REVISÃO SISTEMÁTICA DA LITERATURA
}

\section{PRE-CONSTRUCTION ASSESSMENT MODELS IN SOCIAL HOUSING DEVELOPMENTS: A SYSTEMATIC LITERATURE REVIEW}

\author{
Katrin Rappl i \\ Programa de Pós-Graduação em Arquitetura e Urbanismo, Universidade de São Paulo, São Paulo, SP, Brasil, katrappl@gmail.com \\ Leandro Silva Medrano ii \\ Faculdade de Arquitetura e Urbanismo, Universidade de São Paulo, São Paulo, SP, Brasil, medrano@usp.br
}

\section{Resumo}

O presente estudo realiza uma Revisão Sistemática da Literatura (RSL) para mapear métodos e pesquisas existentes sobre análise e avaliação de projetos habitacionais de interesse social, os quais possam ser aplicados como Avaliação Pré-Construção. O trabalho tem como motivação a constatação amplamente divulgada sobre a falta de qualidade arquitetônica e urbana em empreendimentos de habitação de interesse social, como os realizados pelo Programa Minha Casa Minha Vida, sobretudo na sua primeira e segunda fase. Nesse sentido, busca-se no estudo dos métodos de análise e de avaliação diretrizes que possam contribuir para melhorar a qualidade das relações urbanas nas habitações sociais no país. Destarte, o trabalho realiza uma RSL que tem como base os seguintes indexadores: a plataforma Scielo (Scientific Electronic Library Online), Scopus, Web of Science, Science Direct, o Google Scholar e a Biblioteca Digital Brasileira de Teses e Dissertações. Considerando os resultados, verifica-se que as publicações e estudos sobre esse assunto ainda são escassos na literatura analítica nessa área, de modo geral, faltam referências, protocolos e parâmetros para a avaliação de projetos e sua relação com o espaço urbano antes da etapa de construção, especialmente quando considerado o caso brasileiro. Vê-se, portanto, potencial para o desenvolvimento de pesquisas e do conhecimento metodológico no campo que denominamos como Avaliação Pré-Construção (APC), sobretudo quando considerada a demanda nacional por habitações destinadas à população de menor renda.

Palavras-chave: Avaliação Pré-Construção. Método de avaliação. Método de análise. Habitação de interesse social.

\begin{abstract}
The present study performs a Systematic Review of Literature (RSL) to map existing methods and research on the analysis and evaluation of housing projects of social interest that can be applied as Pre-Construction Evaluation. The work is motivated by a widely publicized finding of the lack of architectural and urban quality in housing developments of social interest, such as those carried out by the Minha Casa Minha Vida Program, especially in its first and second phases. In this sense, we seek to study methods of analysis and evaluation guidelines that can contribute to improving the quality of urban relations in social housing in the country. Thus, the RSL is based on the following reference databases: Scielo (Scientific Electronic Library Online) platform, Scopus, Web of Science, Science Direct and the Brazilian Digital Library of Theses and Dissertations. Results indicate that the publications and studies on this subject are still scarce in the analytical literature in this area. In general, there are no references, protocols, and parameters for the evaluation of projects and their relation with the urban space before the stage of construction, especially when considered this Brazilian case. Therefore, we see a potential for the development of research and methodological knowledge in the field we call the Pre-Construction Assessment (APC), especially when considering the national demand for housing for the lower income population.
\end{abstract}

Keywords: Pre-construction evaluation. Assessment method. Analysis method. Social housing

How to cite this article:

RAPPL, Katrin; MEDRANO, Leandro Silva. Modelos de avaliação pré-construção em empreendimentos habitacionais de interesse social: uma revisão sistemática da literatura. PARC Pesquisa em Arquitetura e Construção, Campinas, SP, v. 8, n. 4, p. 286-300, dez. 2017. ISSN 19806809. Disponível em: <https://periodicos.sbu.unicamp.br/ojs/index.php/parc/article/view/8650238>. Acesso em: 05 mar. 2018. doi: https://doi.org/10.20396/parc.v8i4.8650238. 


\section{Introdução}

Atualmente, grande parte da produção de habitação de interesse social (HIS) no Brasil está vinculada aos resultados da primeira e segunda fase do Programa Minha Casa Minha Vida (PMCMV), lançados pelo Governo Federal em 2009 e 2011 respectivamente. A construção de moradias com o objetivo de diminuir o déficit habitacional foi uma das metas deste programa, assim como o estímulo à economia, através de um modelo de cooperação entre estados, municípios, governo federal e empreendedores.

Até dezembro de 2016, cerca de 4,5 milhões de moradias haviam sido contratadas e 3,2 milhões foram entregues ${ }^{1}$. Apesar do número expressivo de habitações construídas, os resultados em sua maioria não têm garantido projetos democráticos e com qualidade (arquitetônica e urbana), indicando que ainda existem barreiras do ponto de vista da arquitetura e do urbanismo a serem superadas no país.

Por um lado, trata-se de um programa que destacou-se em relação às políticas habitacionais que o antecederam, por considerar as seguintes premissas: reconhecer a questão do déficit habitacional nacional como instrumento metodológico de gestão (BONDUKI, 2008; MARICATO, 2009); inovar em relação às estratégias de financiamento e subsídios; comprometer-se com uma ação articulada em escala nacional; e considerar o atendimento para a faixa de renda mais baixa (faixa 1), a qual, de maneira geral, sempre esteve fora dos programas habitacionais (AMORE; SHIMBO; RUFINO, 2015). Além disso, inovou ao introduzir o subsídio desatrelado do crédito, pois promoveu o acesso ao subsídio e à moradia para as famílias da faixa 1 , independentemente de sua capacidade de contrair crédito (ELOY; COSTA; ROSSETTO, 2013).

Por outro lado, a partir dos resultados da primeira e segunda fase $^{2}$, o programa não parece ter atendido às expectativas (CARDOSO, 2013; KRAUSE; BALBIM; LIMA NETO, 2013; KLINK; DENALDI, 2014). A grande maioria das habitações construídas gera preocupação do ponto de vista da arquitetura e do urbanismo, devido à falta de qualidade e pela pouca integração desses novos aglomerados habitacionais às cidades onde estão localizados. Reforçando, conforme afirma Rufino (2015), uma lógica baseada na "concepção privada de cidade", ou seja, a partir do predomínio do condomínio (privado) e da falta de espaços públicos de sociabilização. É consensual, à crítica especializada, que esses novos bairros surgem no país aparentemente sem preocupação com a qualidade urbana, alinhando modelos padronizados, com baixo padrão construtivo, baixa densidade e monofuncionalidade, independentemente do local de implantação e perfil dos moradores (BONDUKI,
2009; FERREIRA, 2012; AMORE; SHIMBO; RUFINO, 2015).

De modo resumido, as principais críticas ao programa tratam dos seguintes pontos:

- Falta de articulação do programa habitacional com a política urbana (FERREIRA, 2012; ROLNIK et al., 2010; PEQUENO; ROSA, 2015; RAPPL, 2015);

- Localização periférica dos empreendimentos (FERREIRA， 2012; MARQUES; RODRIGUES, 2013; ROLNIK et al., 2014; AMORE, SHIMBO, RUFINO, 2015);

- Possíveis excedentes habitacionais alheios à lógica de demanda efetiva (LOPES; SHIMBO, 2015);

- Baixa qualidade urbana e arquitetônica dos empreendimentos (BONDUKI, 2009; FERREIRA, 2012; ROLNIK et al., 2010; AMORE, SHIMBO, RUFINO, 2015);

- Ausência de avaliação de projetos (RAPPL, 2015; NAGLE; RAPPL; MEDRANO, 2013; KOWALTOWSKI et al., 2015);

- Descontinuidade do programa em relação ao SNHIS (Sistema Nacional de Habitação de Interesse Social) (BONDUKI, 2009; KRAUSE; BALBIM; LIMA NETO, 2013);

- Ausência de concursos de arquitetura e outros mecanismos que poderiam contribuir para o aperfeiçoamento dos projetos (MEDRANO; SPINELLI, 2014; PEREIRA, 2014).

Diante da constatação dessa realidade, torna-se premente a busca por alternativas à falta de qualidade arquitetônica e urbana nos empreendimentos habitacionais contemporâneos no país, questão adotada como motivação desse trabalho. Para isso, recorre-se ao estudo dos métodos com o objetivo de auxiliar na avaliação da produção habitacional e na sua relação com o espaço urbano (cidade), principalmente antes da construção. A finalidade desse tipo de abordagem é nortear os projetos, aprimorar metodologias e soluções projetuais, assim como auxiliar gestores no planejamento e na avaliação dos resultados, de modo a contribuir para o estabelecimento de diretrizes que possibilitem empreendimentos com melhores qualidades.

Nesse sentido, as diversas metodologias qualitativas de análise e avaliação de projetos habitacionais desenvolvidas no campo da Arquitetura e do Urbanismo, buscam encontrar mecanismos com base em teorias, conceitos e práticas para auxiliar o processo crítico e consequentemente melhores resultados. Contudo, esse tipo de abordagem relacionada à qualidade do projeto 
habitacional teve pouca inserção no Brasil (RAPPL, 2015; NAGLE; RAPPL; MEDRANO, 2013).

Por um lado, há que se reconhecer que ao longo dos últimos 16 anos houve avanço nas políticas públicas, nos instrumentos urbanos e na construção de moradias no país (VALENÇA; BONATES, 2010), com a aprovação do Estatuto das Cidades em 2001, a criação do Ministério das Cidades em 2003, a aprovação da Política Nacional de Habitação em 2004, a criação dos Planos Diretores Municipais em 2006 e o lançamento do PMCMV em 2009. Entretanto, os resultados indicam que as ferramentas existentes para avaliar os projetos de habitação ainda são insuficientes para sua aplicação na escala exigida pelos grandes programas nacionais, como o PMCMV.

\section{Avaliação e Crítica em Projetos}

O processo crítico, assim como a aplicação de métodos de avaliação em projetos habitacionais, tem como objetivo proporcionar melhores resultados projetuais e contribuir para a reflexão sobre os aspectos envolvidos. Para diversos autores, a qualidade da arquitetura de um determinado local tem relação direta com a forma que a disciplina estabelece seus parâmetros de avaliação crítica e conceituação. Nesse sentido, o papel da crítica é primordial na busca por uma arquitetura de qualidade (SAUNDERS, 2007), assim como para a construção de conhecimento, pois proporciona oportunidades para o questionamento, reflexão e alteração de critérios que não se encaixam na interpretação do que é um "bom projeto".

Tanto a análise, quanto a avaliação fazem parte desse processo crítico e estão relacionadas com a forma de abordagem (metodologia) do objeto de estudo. A teoria está presente nos dois casos e ao mesmo tempo que norteia, ela também pode ser construída, ou seja, não é fixa (ZEIN, 2001). Já o método é uma base lógica pensada para alcançar uma meta particular (CAMPOS, 1992; VOORDT; WEGEN, 2013), o qual tem seu próprio procedimento metodológico e apoia-se na aplicação de técnicas, ferramentas, instrumentos ou levantamentos específicos (GIL, 2010).

Atualmente, é possível identificar métodos e ferramentas com diferentes amplitudes e profundidades, que podem ser aplicados em diversas fases da "vida" de um edifício (concepção, projeto, construção, uso, reabilitação) e buscam melhorar a qualidade dos projetos e soluções. Alguns são voltados para o apoio aos projetistas e arquitetos, outros são de apoio aos clientes, futuros usuários e ainda de auxílio a gestores. Uns exigem conhecimentos técnicos prévio e são aplicados por profissionais da área (arquitetos, engenheiros, pesquisadores), enquanto outros podem ser executados por qualquer pessoa (KERNAHAN et al., 1992). Apesar dessas diferenças, isso não implica necessariamente que cada ferramenta auxilie apenas um público e seja utilizada em apenas uma etapa do projeto, muitas delas abrangem mais de um público alvo e podem ser utilizadas em mais de uma fase.

Além disso, esses termos podem ser empregados em distintos contextos: para a análise ou avaliação do produto, de projetos ou de parte dele (conhecido como: "avaliação do produto"); ou para a avaliação do processo de projeto, de gerenciamento e construção (conhecido como: "avaliação do processo") (VOORDT; WEGEN, 2013).

Do ponto de vista da "avaliação do produto", o termo "análise" se refere à descrição de um projeto, de seus dados, formas, questões colocadas pela obra e suas soluções resultantes, ou seja, é um exame da cada parte constituinte do projeto (do todo). Já o termo "avaliação" vem da área financeira, na qual está relacionado com o valor do dinheiro e ao cálculo do câmbio e significa determinar o valor ou estipular quanto alguma coisa vale (VOORDT; WEGEN, 2013). Em arquitetura, a "avaliação" trata da atribuição de valor aos conceitos avaliados, com a finalidade de proporcionar $\mathrm{o}$ reconhecimento ou a desvalorização de determinada produção, para a ponderação de soluções, ou até para a possível comparação entre diferentes projetos.

Do ponto de vista do "processo de projeto", o próprio ato de decidir durante o desenvolvimento de um projeto (processos de análise, síntese e avaliação) pode ser considerado um tipo de julgamento (KOWALTOWSKI et al., 2013). Nesse sentido, o processo de "análise" envolve a descrição do problema e a listagem de todos os requisitos de projeto, a partir da coleta de informações e ideias, bem como a classificação dos objetivos e necessidades (conjunto de especificações logicamente relacionadas), os quais vão auxiliar na ordenação, estruturação do problema e consequentemente na solução projetual (JONES, 1984; LAWSON, 1997; CARMONA et al., 2003; VOORDT; WEGEN, 2013). Já a "avaliação" busca determinar se as soluções e resultados (totais ou parciais) apresentados satisfazem objetivos, requisitos ou necessidades estabelecidas anteriormente. Ou seja, pode ser medida em contraposição às metas anteriores (JONES, 1984; LAWSON, 1997; CARMONA et al., 2003; VOORDT; WEGEN, 2013).

Além da distinção entre avaliações voltadas para 0 "processo" ou para o "produto", é possível encontrar também a classificação entre "ex ante" (antes da realização ou do fato) ou "ex post" (depois da realização ou do fato) (VOORDT; WEGEN, 2013). Nesse sentido, dentro das "avaliações para o produto", uma Avaliação "Ex Ante" (AEA) ocorre, por exemplo, antes da construção da edificação. 
Neste artigo, sugerimos a criação da nomenclatura Avaliação Pré-Construção (APC), pois nos pareceu importante definir uma etapa de avaliação do projeto de arquitetura distinta da contemplada pelo termo AEA, que é muito centrada às "avaliações voltadas para o produto". A nomenclatura e sigla APC também foi sugerida pela relação de semelhança com o termo Avaliação Pós-Ocupação (APO), amplamente conhecido na literatura da área, mas que indica processos de avaliação realizados após a construção e ocupação de edificações. Assim, consideramos a APC como uma etapa a ser realizada antes do início da construção, para avaliar seus impactos e a possibilidade da edificação atingir seus objetivos práticos e sociais. Alguns exemplos de APC são: a avaliação de um projeto em relação às especificações do programa de necessidades; a avaliação do próprio programa de necessidades, na qual pode-se verificar se ele está de acordo com os requisitos dos clientes, diretrizes de qualidade, com o orçamento, normas e outras exigências; e também o relatório ambiental, no qual se examina a probabilidade de impacto do projeto sobre o ambiente (VOORDT; WEGEN, 2013).

No contexto da produção habitacional brasileira, o ideal é que esse tipo de avaliação APC possa ser aplicado antes da decisão de construir um edifício, entre as etapas de projeto ("produto") e de construção. O intuito é constatar se o projeto atende ou não aos parâmetros elencados como essenciais, de forma a evitar problemas "conhecidos" antes de sua edificação (em uma etapa menos custosa), certificar que o mesmo tem probabilidade de sucesso e pode contribuir para a construção de cidades com qualidade.

Já a avaliação "ex post" é realizada depois que a edificação já está pronta ou em uso. Ela determina se as expectativas e metas elencadas inicialmente foram atingidas, um exemplo desse tipo de avaliação é a Avaliação Pós-Ocupação (APO). As APOs também são importantes, pois permitem identificar problemas que não eram previsíveis e que agora podem ser evitados, mais precisamente, pela aplicação dessas constatações como diretrizes de projeto (no processo de projeto) ou como parâmetros de Avaliação Pré-Construção (APC).

Assim, as avaliações permitem indicar questões que podem induzir ao aprimoramento de projetos ("processo" e "produto"), contribuir para a formação de novas teorias, desenvolver novas ferramentas e também revelar fenômenos imprevistos e não intencionais, positivos ou negativos (VOORDT; WEGEN, 2013).

Tendo em vista que uma avaliação pode existir por diversas razões, objetivos, naturezas, se diferenciar em abrangência, método, tempo de avaliação, ter diferentes parâmetros e focar em diversos públicos-alvo
(KOWALTOWSKI et al., 2013), é importante saber o que será avaliado, assim como de quais premissas (base teórica) deverão partir os métodos, antes mesmo do início da avaliação. As perguntas chaves são: Por que será avaliado?; Como será?; Quando?; Para quem e por quem? (MONTANER, 2007; VOORDT; WEGEN, 2013; KOWALTOWSKI et al., 2013).

Os resultados de uma pesquisa de avaliação podem ser apresentados sob a forma de listas de verificação, selos de aprovação, certificados e manuais (VOORDT; WEGEN, 2013). Essas ferramentas são adequadas pois auxiliam na verificação da qualidade de projetos, no desenvolvimento de teorias, orientam e direcionam normas, códigos, leis e até mesmo políticas públicas. Dessa forma, é de extrema importância o emprego desses métodos, assim como a divulgação e o fácil acesso aos seus resultados.

Nesse contexto, a necessidade de verificar se um projeto ("produto") se enquadra nos objetivos e atributos de um edifício com qualidade, (a partir da aplicação de procedimentos metodológicos), levou a identificação e mapeamento das principais pesquisas e métodos de análise e de avaliação que possam ser aplicados como avaliação APC para projetos de HIS.

\section{Metodologia}

A busca para identificar os principais estudos e métodos sobre análise e avaliação de projetos ("produto") APC na área habitacional teve início com uma revisão sistemática da literatura (RSL). O objetivo da revisão foi construir um panorama das publicações existentes, através do mapeamento e identificação das principais pesquisas relacionadas ao tema, de forma a responder a pergunta: "O que existe na área habitacional em termos de pesquisas e métodos de avaliação APC?", assim como indicar lacunas a preencher e possibilidades de abordagens para o desenvolvimento do conhecimento sobre esse assunto.

A estratégia de busca, conforme indica Dresch, Lacerda e Antunes Jr. (2015), foi escolhida procurando evitar viés e aumentar as chances de localizar os principais estudos relevantes sobre o tema. Sendo assim, adotou-se os termos a serem pesquisados em diferentes idiomas (inglês, espanhol e português), além da utilização de sinônimos e de combinação de palavras-chave, variando de acordo com a plataforma de busca. Também foram considerados diferentes locais de pesquisa, alguns mais específicos, como por exemplo, determinadas revistas Q1 e outros mais gerais, como o Google Scholar.

A revisão adotou o período de 2005 a 2015 e utilizou os seguintes termos de busca em títulos, resumo e palavraschave $^{3}$ : 
a) Inglês: evaluation method; assessment method; analysis method; method of evaluation; method of assessment; method of analysis; evaluation protocol; quality indicator; evaluation methodology; qualitative analysis; quality perception; critical analysis; ex ante assessment; ex ante analysis;

b) Espanhol: método de evaluación; método de análisis; protocolo de evaluación; indicador de calidad; metodología de evaluación; análisis cualitativo; percepción de la calidad; análisis critico; evaluación ex ante; análisis ex ante;

c) Português: método de avaliação; método de análise; protocolo de avaliação; indicador de qualidade; metodologia de avaliação; análise qualitativa; percepção de qualidade; análise crítica; avaliação ex ante; análise ex ante.

Considerando que os resultados de algumas buscas com essas palavras-chave isoladas foram demasiadamente amplos, optou-se por uma melhor especificação dos termos - mais focada na questão das HIS. Para tanto, as mesmas palavras-chave foram utilizadas (em inglês, português e espanhol) e acrescidas das palavras: social housing, (em inglês); vivienda social e vivienda protegida, (em espanhol); e habitação social, (em português). Dessa forma, foram pesquisados ${ }^{4} 44$ conjuntos de palavras-chave em alguns casos.

Esses termos foram procurados em diferentes locais de busca, elencados na sequência:

1. 121 revistas selecionadas: essa busca utilizou como referência o fator de impacto SJR (Scientific Journal Ranking), mais precisamente o índice de 2014. A partir desse índice, foram selecionadas todas as revistas classificadas como Q1 nas categorias relacionadas à arquitetura, são elas: Architecture (21 revistas); Urban Studies (33 revistas); Nature and Landscape Conservation (29 revistas); Building and Construction (38 revistas). Nessa etapa foram utilizados os termos de busca apenas em inglês, visto que a categoria das revistas focava a área de interesse. No total, 56 trabalhos foram selecionados nas revistas pesquisadas 5 .

2. Plataformas de dados Scielo (Scientific Electronic Library Online) e Scopus: nessa etapa, além das palavras-chave de busca em inglês, foi necessário utilizar também nos idiomas espanhol e português. No total, 8 trabalhos foram selecionados (1 da Scielo e 7 da Scopus).

3. Google Scholar: essa etapa procurou encontrar métodos de análise e avaliação na área habitacional que não estivessem citados necessariamente em artigos, congressos, livros, teses e dissertações, mas em manuais, relatórios de sites governamentais ou outro tipo de material. Ademais, buscou-se por documentos que levassem a sites de instituições que estudam esse assunto. Devido ao grande número de resultados encontrados para cada palavra-chave, foi necessário focar a área de interesse da pesquisa e por isso foram utilizados os 44 conjuntos de termos de busca, nos três idiomas. Do total de trabalhos inicialmente selecionados, 45 contemplam a tabela final.

4. Plataformas de dados Web of Science e Science Direct: nessa etapa foram utilizados os 14 conjuntos de palavras-chave em inglês. As publicações foram analisadas e após descartar trabalhos que já haviam sido identificados nas buscas anteriores, selecionaram-se 5 da base Web of Science e 3 da Science Direct.

5. Biblioteca Digital Brasileira de Teses e Dissertações: essa busca utilizou os 44 conjuntos de termos nos três idiomas. Após uma análise mais detalhada da versão completa dos trabalhos e a eliminação daqueles já identificados anteriormente, selecionaram-se 3 .

Inicialmente, as publicações foram identificadas pelos títulos, resumos e palavras-chave e posteriormente, após uma análise mais cuidadosa da versão completa de cada trabalho, alguns foram descartados e outros selecionados. Os critérios para a inclusão e a exclusão de estudos na revisão levaram em consideração a relação entre arquitetura (habitação) e cidade (dimensão urbana). Durante o levantamento, evitou-se selecionar pesquisas com abordagens estritamente técnicas, voltadas aos desempenhos dos sistemas construtivos, conforto térmico, acústico, desempenho lumínico e eficiência energética, assim como análises apenas da unidade habitacional, sem considerar aspectos do edifício ou do entorno. Essa opção foi devido à necessidade de alinhar a RSL às intenções dessa pesquisa, mais voltada às relações urbanas das HIS projetadas no Brasil. Nesse sentido, foi dado ênfase às avaliações de projetos e aos estudos que tratam da relação entre o edifício e o espaço urbano. Da mesma forma, foram selecionados métodos e pesquisas de sustentabilidade ${ }^{6}$ que venham a contribuir para esta questão (dimensão urbana e habitação) e que, mesmo parcialmente, possam ser utilizados com uma abordagem ex ante.

A extensão da busca procurou encontrar a maior quantidade possível de estudos relevantes dentro dos termos definidos na metodologia indicada. Contudo, salientamos que a amostragem pode ter excluído trabalhos relevantes que não foram publicados em revistas de alto fator de impacto e reconhecimento acadêmico internacional. Destarte, os resultados aqui 
apresentados podem não corresponder ao "estado da arte" dos temas em tela, mas procuram relatar e discutir as pesquisas de maior impacto segundo sistemas internacionais de divulgação científica.

\section{Síntese dos Resultados}

No total, foram selecionados 120 trabalhos na revisão sistemática realizada, apresentados no gráfico 1 por ano de publicação.

Os dados indicam que a quantidade de resultados para o período de busca (2005-2015) sobre o tema avaliação de projetos habitacionais (APC) é pequena (Gráfico 1), com uma média de 12 publicações por ano. Essa constatação é ainda mais perceptível se for comparada com outras áreas do conhecimento mais consolidadas, que têm métodos específicos desenvolvidos, como das avaliações ex post voltadas para as avaliações de desempenho ambiental (eficiência energética), de conforto (sonoro, acústico, térmico, lumínico), por exemplo.

Entre os tipos de documentos encontrados na pesquisa, observa-se a predominância do número de artigos, em torno de 6 vezes maior do que a quantidade de livros, teses e dissertações (Gráfico 2). Sugerindo que a principal forma de divulgação dos estudos nesse campo está sendo realizada através das revistas científicas.
Também é notável a diferença entre a quantidade de artigos sobre esse assunto em publicações nacionais e internacionais (Gráfico 2). A maior parte delas é externa ao Brasil, indicando a necessidade do aprofundamento dessa discussão em cenário nacional.

A tabela 1 apresenta os principais termos identificados nos títulos dos trabalhos selecionados na RSL. Eles foram agrupados de acordo com a semelhança entre seus significados e a separação em 7 grupos buscou facilitar a compreensão dos resultados, visto que alguns termos poderiam ser apresentados em mais de um grupo, por exemplo: "design methods" (grupo 1 ou 5), "parâmetro de qualidade" (grupo 4 ou 6) e "quality indicador" (grupo 4 ou 6), justamente pela inter-relação entre eles.

Durante esse processo, constatou-se a variedade de palavras utilizadas para o mesmo significado nos estudos, por exemplo, termos como: "ferramentas" e "instrumentos"; "parâmetros" e "indicadores" (grupo 4), os quais muitas vezes acabam aparecendo com o mesmo sentido. Fato confirmado também por outros autores, os quais indicam que no uso cotidiano, termos como, "características", "propriedades" e "atributos" acabam sendo substituídos pelas palavras "qualidade" e "valor" de projeto (GERRITSE, 2008; MACMILLAN, 2006; PULTAR, 1996; THOMSON et al., 2003; VOORDT; WEGEN, 2013; VOLKER, 2010).

Gráfico 1 - Número de artigos, teses, dissertações, artigos de congressos e outros documentos (relatórios, manuais) encontrados na revisão por ano

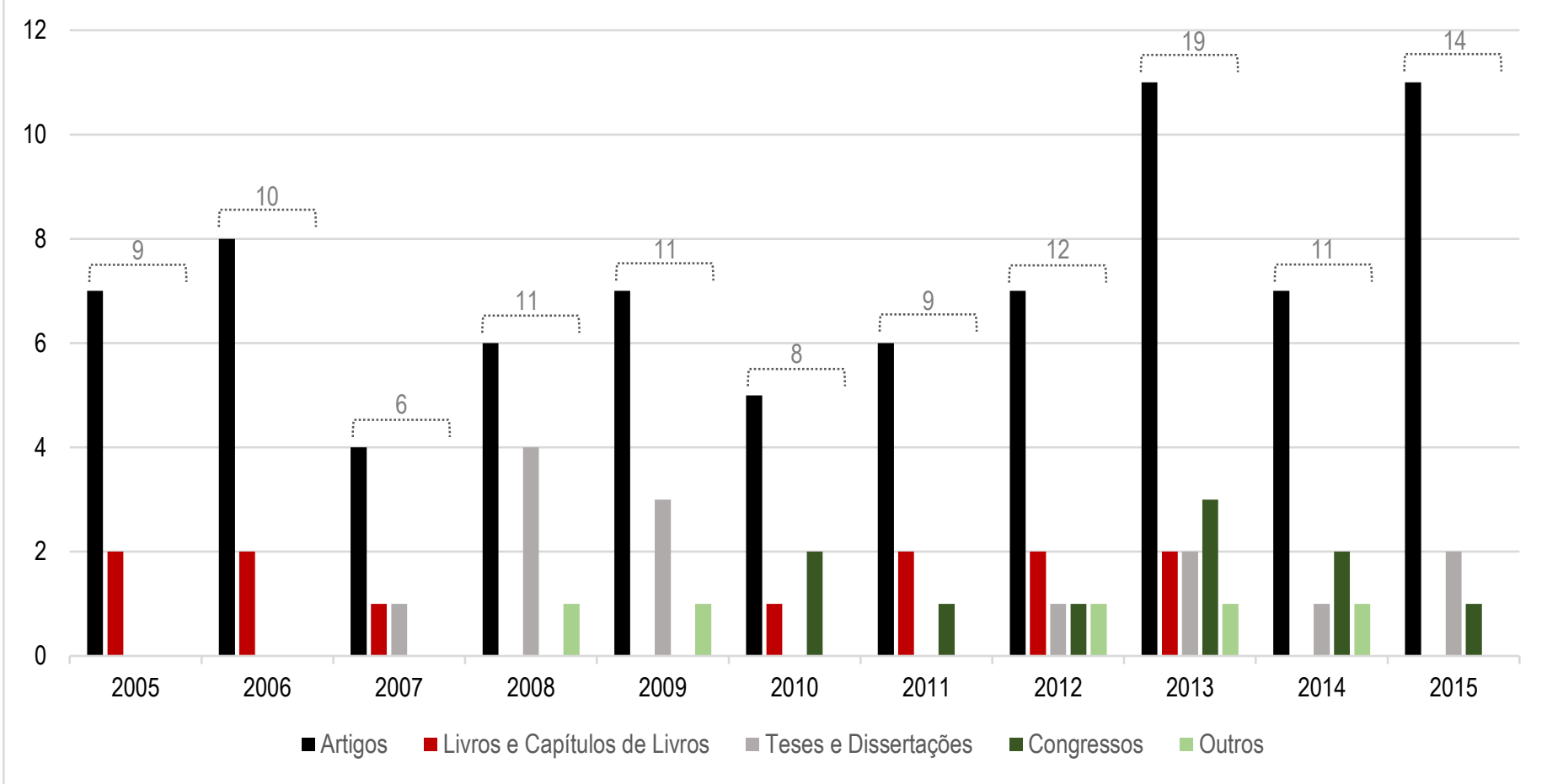

Fonte: Os autores. 
Gráfico 2 - Número de publicações nacionais e internacionais encontradas na revisão separadas por tipo

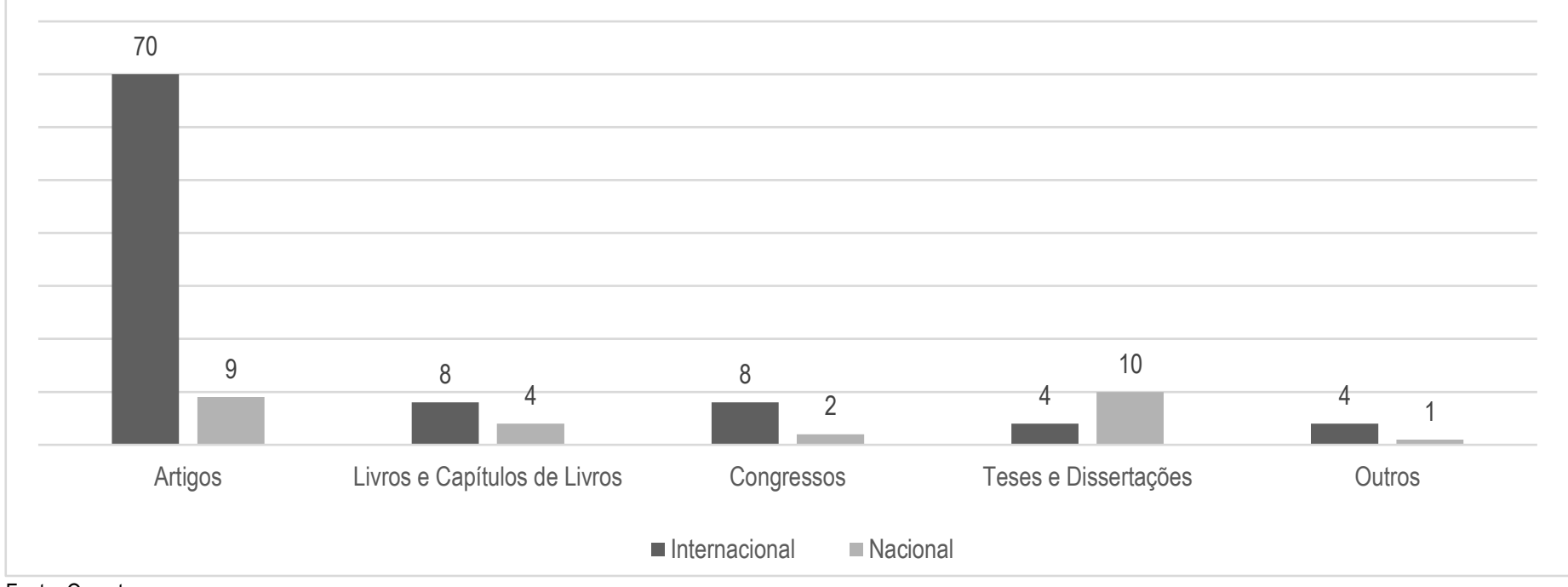

Fonte: Os autores.

Gráfico 3 - Número de publicações selecionadas por cada conjunto de palavras-chave nos três idiomas?

2.) Avaliação ex ante; Ex ante assessment; Evaluación ex ante

3.) Percepção de qualidade; Quality perception; Percepción de la calidad

4.) Protocolo de avaliação; Evaluation protocol; Protocolo de evaluación

5.) Análise qualitativa; Qualitative analysis; Análisis cualitativo

6.) Metodologia de avaliação; Evaluation methodology; Metodología de evaluación

7.) Análise crítica; Critical analysis; Análisis critico

8.) Método de análise; Analysis method; Method of analysis; Método de análisis

9.) Indicador de qualidade; Quality indicator; Indicador de calidad

10.) Método de avaliação; Evaluation method; Assessment method; Method of evaluation; Method of assessment; Método de evaluación

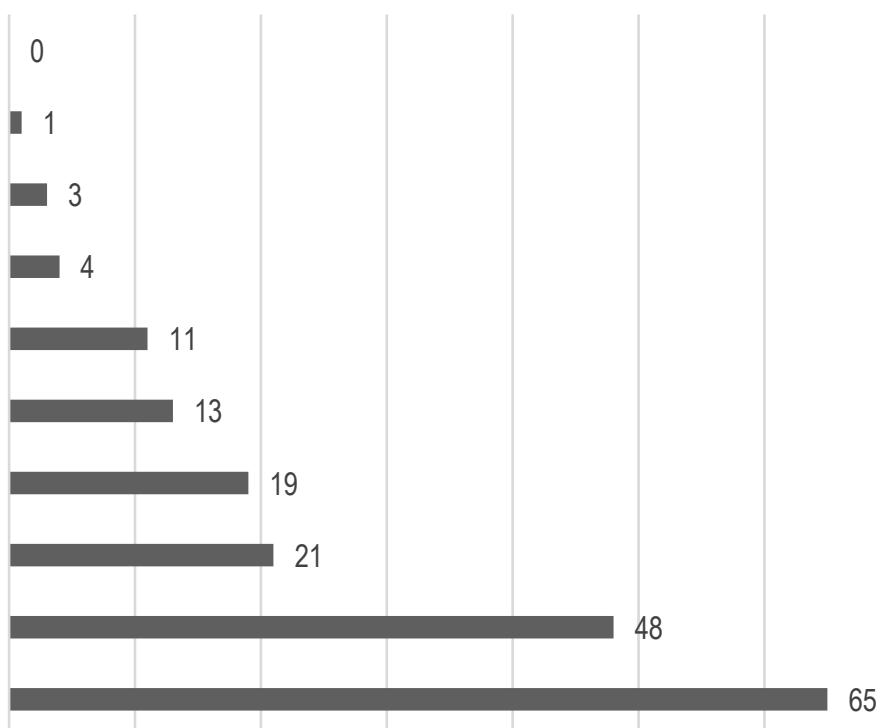

Fonte: Os autores.

As informações da tabela 1, juntamente com os dados do gráfico 3 sobre o número de publicações levantadas para cada conjunto de palavras-chave, indicam a predominância de trabalhos que tratam de indicadores de qualidade e métodos de avaliação sobre os temas de sustentabilidade e da área ambiental (grupo 3 - Tabela 1) e (conjuntos 9 e 10 - Gráfico 3). Voltadas principalmente para pesquisas relacionadas aos métodos atuais de certificação de sustentabilidade para edificações, tais como, o LEED (Leadership in Energy and Environmental Design), o BREEAM (Building Research Establishment Environmental Assessment Method), o VERDE ESPAÑA, o SBTool (Sustainable Building Tool), entre outros.
Também se verifica entre os resultados da pesquisa a presença de trabalhos com abordagens relacionadas à qualidade do projeto, do edifício e do espaço construído (grupo 6 da Tabela 1).

A partir dos resultados da amostra, observa-se que alguns métodos de avaliação são específicos para uma fase da "vida" de um edifício, para um público alvo, tipologia e até determinada localidade, enquanto outros são mais gerais e abrangem diversas etapas do ciclo de "vida", várias localidades e tipologias. Alguns são utilizados para certificações e classificações, outros são para estudos de análise e sistematização de dados. 
Tabela 1 - Análise das principais palavras que aparecem nos títulos das pesquisas encontradas na revisão, agrupadas em: Grupo 1 (edifício e projeto), Grupo 2 (avaliação, análise e crítica), Grupo 3 (sustentabilidade e área ambiental), Grupo 4 (diretrizes, indicadores, ferramentas e parâmetros), Grupo 5 (métodos e metodologias), Grupo 6 (qualidade e valor) e Grupo 7 (meio urbano)

\section{Termos e grupos de significados}

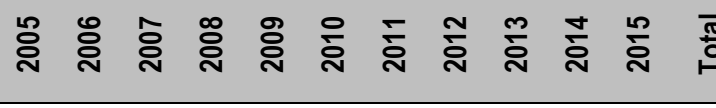

Building assessment 2 1

Design critiquing

Design evaluation

Design knowledge

을 Design method

일 Design moves

Design objectives

Design thinking

Design process

Avaliação; Avaliar; Assessment; Assessing; Evaluate; Evaluation Evaluating;

Evaluación; Evaluar

Análise; Analysis

$\sim$ Análisis comparativo; Comparative analysis

Onálise quantitativa; Quantitative analysis

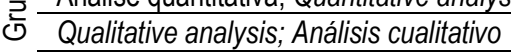

Critical review

Judgements

Preferências dos usuários; Residential satisfaction; Resident perspectives

Ambiente; Ambiental; Environment; Environmental

$m$ Certificação; Certification; Labelling system

을 Grading system; Rating; Rating system

ธั Performance assessment; Performance evaluation; Performance

Sustentabilidade; Sustainability; Sustainable; Sostenible

Checklist

Design guideline

Modelo; Models

Index

Indicador; Indicator

ナ Instrumento; Tool; Ferramenta; Herramienta

은 Instrumento de análisis

Evaluation tool; Assessing instrument

Parâmetro de qualidade

Schemes

Requisitos

Sistema; System

Método de análise

L Método de avaliação; Métodos de evaluación; Evaluation method; Assessment

은 method

Metodologia; Methodology

Qualidade; Quality; Qualities;

Qualificação do espaço; Calidad del espacio

Qualidade de projeto; Design quality

$\odot$ Architectural qualities

Qualidade do edifício

Quality indicator

Qualidade de vida; Quality of life; Life quality

Best practices

Valor; Value

Desenho urbano; Urban Design

$\sim$ Urban patterns

Urbanism

을 Áreas urbanas

Inserção urbana

Urbano; Urbana; Urban

Fonte: Os autores.

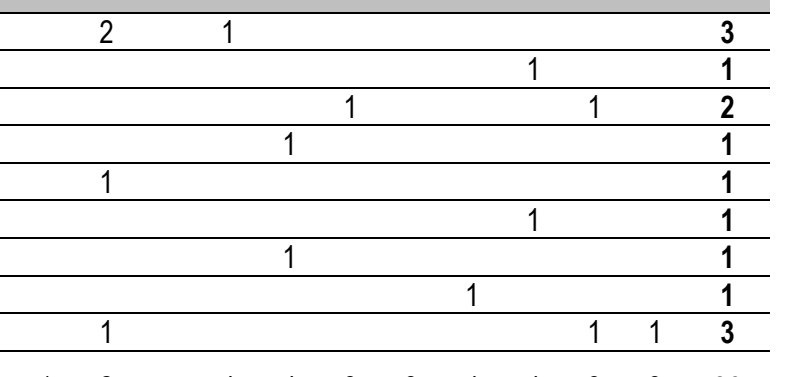


pelo grupo DEGW para o governo do Reino Unido (Communities and Local Government) em 1998, o qual teve destaque na introdução da avaliação habitacional com base na qualidade e não apenas no custo. Além dele, vale a pena ressaltar o método Suíço SEL (Système d'évaluation de logements), inicialmente de 1975, que estabelece parâmetros acima dos valores mínimos presentes na norma oficial do país (SOUSA, 1994). E também o método DQI, desenvolvido em 2003 pelo Conselho da Indústria da Construção (Construction Industry Council - CIC), o qual considera que a qualidade é um aspecto difícil e incerto a medir, mas que o desenvolvimento de ferramentas para aferir e discutir a qualidade do projeto entre os diferentes públicos envolvidos pode ser benéfica (GANN; SALTER; WHYTE, 2003; GANN; WHYTE, 2003; PRASAD, 2004; WHYTE; GANN, 2003).

De modo geral, observa-se entre as pesquisas e métodos da RSL que não existe um consenso sobre como avaliar projetos habitacionais. Visto que uma avaliação pode tratar de diferentes dimensões, escalas de aproximação e etapas do projeto, bem como considerar aspectos tangíveis (abordagem objetiva) e/ou intangíveis (abordagem subjetiva), os quais implicam, necessariamente em formas distintas de aferir.

Assim, a partir dos resultados e juntamente com a fundamentação de Jones (1984), é possível verificar várias formas de avaliar um projeto arquitetônico:

1) Por requerimentos e especificações - o projeto é avaliado em relação às metas descritas anteriormente, normas, especificações, programa de necessidades e até diretrizes. A maioria das pesquisas e métodos de certificação de sustentabilidade se enquadra nesse perfil (Grupo 3), tais como, o BREEAM, o LEED, entre outros.

2) Por julgamento de valor (lógico e por experiência) - esse tipo de avaliação utiliza aspectos das características tangíveis e intangíveis. Pode-se dizer que é uma avaliação mais complexa, por abordar questões subjetivas, por isso exige conhecimento técnico e experiência na área. $\mathrm{O}$ método inglês DQI (Design Quality Indicator) é um exemplo que possui esse tipo de abordagem.

3) Por comparação - é avaliado em relação a outros projetos. Verifica-se, por exemplo, qual é o edifício mais alto ou mais baixo, qual tem maior ou menor densidade, sem de fato mensurar e especificar qual seria a definição "ideal" para esses parâmetros. Um exemplo desse item é o método espanhol I+D+VS (Investigación,
Desarrollo y Vivienda Social), o qual apresenta gráficos com esse tipo de comparação entre os projetos de estudo.

Do mesmo modo, também não há consenso entre os resultados da RSL na forma pela qual os estudos estabelecem seus parâmetros de análise ou avaliação. Nesse sentido, é possível identificar diferentes maneiras, sendo que uma não exclui necessariamente a outra, são elas: a) a partir de conceitos e princípios presentes na literatura da área; b) em diretrizes normativas; c) no estudo de indicadores e grades conceituais de outros métodos de avaliação; d) nas diretrizes e parâmetros utilizados em concursos de projeto; e) a partir de informações relacionadas à satisfação dos moradores e em pesquisas de $\mathrm{APO}$; f) pela observação e constatação de problemas "comuns" na qualidade habitacional; g) através da adaptação de boas práticas e de projetos tidos como exemplares na área.

Com relação às bases conceituais envolvidas nos parâmetros de avaliação desses estudos, a maior parte deles utiliza princípios do Smart Growth (maioria das avaliações ambientais) e ainda do New Urbanism. Notase também, porém em menor quantidade, a presença de trabalhos com parâmetros relacionados ao Desenho Urbano, à Tríade Vitruviana ("utilitas", "venustas" e "firmitas"), à Gramática da Forma (tipo analítica) e à área Ambiente-Comportamento, com aspectos relacionados à psicologia.

Do ponto de vista da relação entre o edifício habitacional com o espaço urbano, a maioria dos estudos abordam as questões do entorno a partir dos seguintes parâmetros: 1) Distância do edifício ao transporte público; 2) Distância entre o edifício e escolas, comércios, serviços e espaços de lazer; 3) Presença de ciclovias no entorno ou espaços para as bicicletas no lote. Esses parâmetros estão relacionados sobretudo aos princípios do Smart Growth e aos objetivos de construir espaços mais compactos e menos dependentes do automóvel.

Contudo, outros aspectos também estão relacionados à qualidade dos espaços habitacionais e urbanos, tais como densidade, usos mistos, diversidade no lote, conexões, fachada ativa, permeabilidade urbana, relação entre o espaço público e privado e aspectos morfológicos relacionados ao entorno ou à malha urbana. Isto é, questões ligadas à configuração dos espaços físicos e aos princípios do Desenho Urbano. No entanto, os dados indicam que entre os exemplos estudados são poucas as pesquisas e métodos que de fato incorporam esses aspectos em seus parâmetros de avaliação (grupo 7 Tabela 1). Esse número é menor ainda se considerarmos as avaliações realizadas antes da etapa de construção. 
Nesse sentido, é necessário o aprofundamento de pesquisas sobre parâmetros que tratam das relações urbanas das HIS, assim como a ampliação de avaliações ex ante. Apesar disso, já é possível notar que pesquisas como a "Ferramenta de Avaliação de Inserção Urbana para Empreendimentos do MCMV" (ROLNIK et al., 2014), os "Parâmetros de Qualidade Urbanística e Arquitetônica" (FERREIRA, 2012) e os "Instrumentos de Avaliação de Projetos" (DINIZ; FEDELI, 2012), possuem diretrizes com grande potencial para serem incorporadas em APC de HIS no Brasil, especialmente no caso do PMCMV. Posto que, seus parâmetros de avaliação consideram a relação entre as habitações e o planejamento urbano, campo que tem muito a contribuir para a integração entre a arquitetura e a cidade.

\section{Discussão}

No Brasil, as preocupações e a busca pelo aumento da qualidade habitacional tiveram origem na década de 90 . Foram criados programas e mecanismos de controle de qualidade em diversas esferas, com o objetivo de evitar problemas constatados, tais como: o Programa da Qualidade da Construção Habitacional do Estado de São Paulo (QUALIHAB) ${ }^{8}$ em 1996; o Programa Brasileiro de Qualidade de Produtividade no Habitat $(\mathrm{PBQP}-\mathrm{H})^{9} \mathrm{em}$ 1998 a nível federal; o Programa Nacional da Habitação (PRONATH) em 1991; e o Programa de Tecnologia da Habitação (Habitare) em 1994, financiado pela FINEP (Financiadora de Estudos e Projetos). Ambos os dois últimos com o objetivo de buscar soluções tecnológicas para os problemas habitacionais brasileiros (GALVÃO; ORNSTEIN; ONO, 2013).

Também se encaixa nesse processo a criação da norma de desempenho mínimo para edifícios habitacionais, a NBR 15.575 (ABNT, 2013) publicada em 2008, a qual especifica critérios e requisitos de qualidade que devem ser atendidos em edifícios de apartamentos, tanto no processo de projeto, quanto na especificação. Assim como, o recente surgimento dos processos de certificação brasileiro, tais como: o Selo Casa Azul (2010) e o Método AQUA-HQE (2008).

Embora esses esforços ofereçam grande potencial para o debate sobre a qualidade dos projetos, os resultados da produção habitacional observados no país levam a crer que essa discussão ainda necessita progredir, garantir a continuidade, incorporar processos avaliativos e acúmulo de conhecimento crítico, visto que a avaliação sistemática na área de HIS parece estar ausente.

Nesse sentido, a lacuna detectada nas pesquisas relacionadas às APCs dedicadas ao tema das HIS, considerado o período em análise e a metodologia RSL implementada no trabalho, revelam uma relação correlata com os resultados do PMCMV - amplamente criticado pela literatura analítica da área. Verifica-se que a implementação da APC no fluxo operacional de programas como o PMCMV seria um grande avanço para as políticas urbanas nacionais, pois permitiria uma maior discussão qualitativa em relação aos projetos habitacionais - o que daria margem, inclusive, às inovações tecnológicas, programáticas, urbanísticas e tipológicas. Ademais, o mecanismo permitiria maior evidência à função social e à democratização do processo, pois colocaria o projeto de arquitetura e as questões urbanas na pauta das discussões cotidianas das cidades - o que poderia aproximar o habitat às questões da cidadania, geografia social, inclusão territorial etc. Como exemplo prático, foi elaborado um gráfico complementar ao Fluxo operacional do PMCMV atualmente utilizado pela Caixa Econômica Federal (Figura 1), com a sugestão de implementação da APC. Nota-se que além da APC, propomos uma etapa de "consulta à população", para os casos onde esse procedimento for pertinente.

Esse processo não descarta a importância e a necessidade da utilização de outros "sistemas de suporte" utilizados durante o processo de projeto ou em avaliações ex post.

\section{Conclusão}

Desde o estudo realizado, pode-se constatar que apesar da importância dos trabalhos acadêmicos relacionados à qualidade do projeto arquitetônico e urbano na construção de HIS no país, ainda são poucas as pesquisas que abordam esse tema. O número reduzido de resultados na RSL indica a necessidade de um número maior de pesquisas que avaliem a relação entre a qualidade arquitetônica e urbana, em empreendimentos habitacionais, antes da construção. Principalmente quando se considera as especificidades do caso brasileiro e/ou dos países em desenvolvimento.

Nota-se também que as publicações relacionadas à avaliação da qualidade do projeto de HIS dedicam-se, prioritariamente, à análise de obras construídas (estudos de APO ou similares). Não descartamos a relevância desse tipo de pesquisa, pois são fundamentais para que os equívocos de um determinado projeto não se repitam, bem como para que suas boas soluções possam ser replicadas em contextos adequados. Entretanto, quando o tema são os programas governamentais de grande escala, como o PMCMV, o retorno pode não corresponder às necessidades da demanda. Nesses casos, a APC poderia tornar-se um instrumento essencial à qualidade do projeto e da política habitacional como um todo. Assim, verificase potencial para melhorar a qualidade das HIS no Brasil a partir da aplicação de modelos de avaliação APC antes da construção do projeto, exemplificado no esquema da Figura 1. 
Figura 1 - Fluxo operacional complementar ao PMCMV para construtoras (em vermelho). Considerando habitação para as famílias da faixa 1 de renda

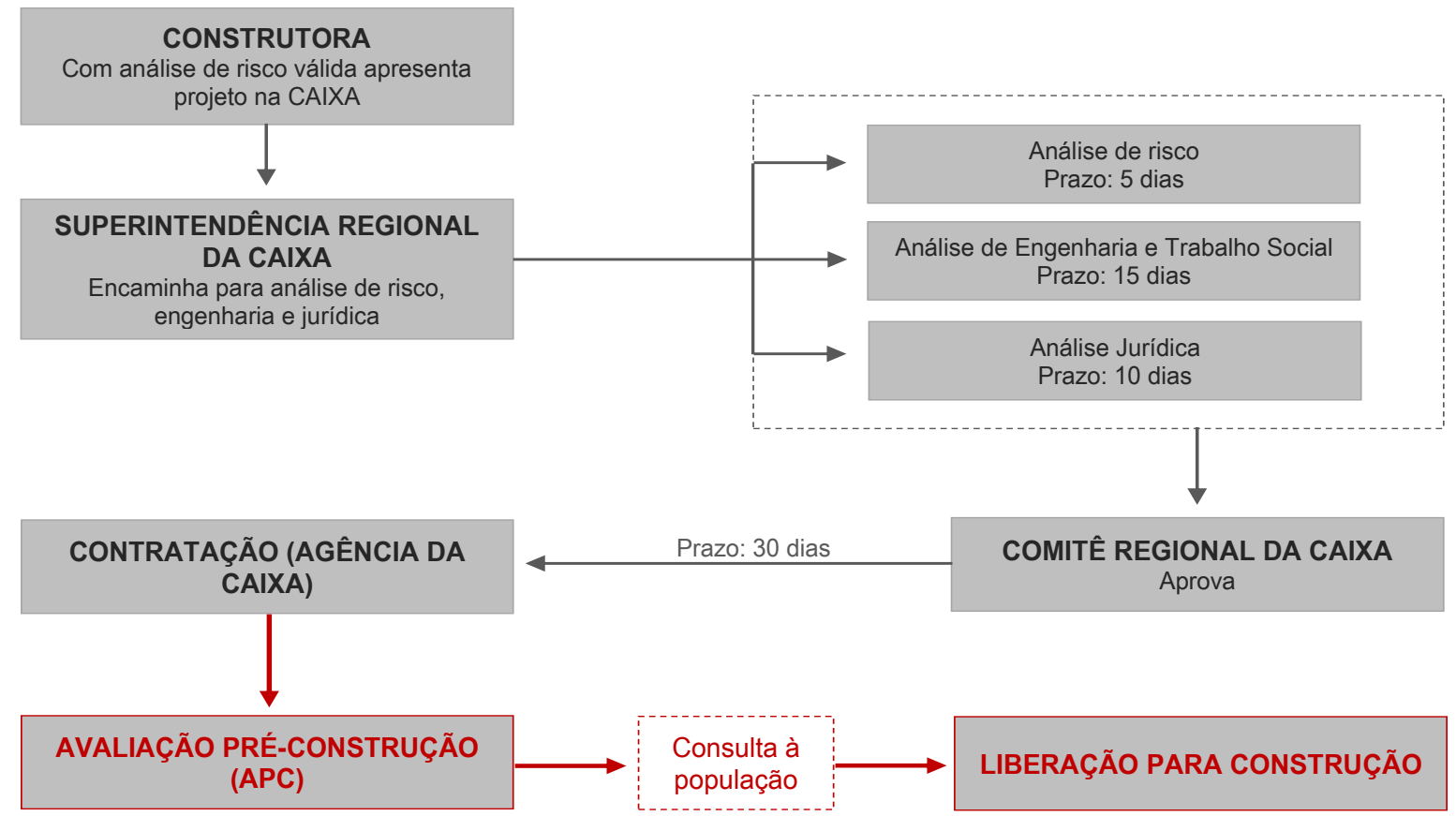

Fonte: Adaptado pelos autores a partir do site da CAIXA [http://www.caixa.gov.br/voce/habitacao/minha-casa-minha-vida/urbana/Paginas/default.aspx]

É oportuno salientar que a metodologia da RSL aqui apresentada, em relação aos periódicos, teve como foco as publicações indexadas e de alto impacto na área. Tal amostragem pode apresentar distorções em relação à quantidade e relevância dos exemplos analisados quando comparados com o que efetivamente foi produzido na área. Contudo, no que diz respeito aos padrões atualmente evidenciados no meio acadêmico, revela um panorama circunstanciado nos padrões de excelência dos indexadores mais respeitados da área. $\mathrm{O}$ que deve aproximar, pelo menos em termos do impacto dos trabalhos publicados, a amostragem da realidade.

Em suma, a revisão sugere que o campo das avaliações APC de projetos habitacionais ainda é uma área com pouca publicação sobre o assunto, verifica-se também que faltam referências, protocolos e métodos na área da arquitetura e do urbanismo do que seriam considerados projetos de boa qualidade. Por exemplo, observam-se diretrizes e até mesmo avaliações que muitas vezes não deixam claro os parâmetros de referência utilizados. Vêse, portanto a necessidade do desenvolvimento de pesquisas e do conhecimento sobre esse tema, assim como o aprofundamento sobre métodos existentes (tanto de análise quanto de avaliação de projetos), especialmente quando levado em consideração a realidade habitacional nacional e a falta de avaliação dos projetos derivados das políticas de habitação.

Por fim, os resultados desse artigo também indicam a necessidade do aprofundamento na discussão sobre parâmetros de qualidade - que abordem as relações urbanas das HIS - os quais possam ser adaptados e aplicados em avaliações APC para a realidade habitacional brasileira.

\section{Agradecimentos}

Agradecimentos são expressos à FAPESP (Processo número 2015/03676-0) e ao CNPq (Bolsa de Produtividade).

\section{Notas}

(1) Dados referentes ao $4^{\circ}$ Balanço do PAC (2015-2018) (MINISTÉRIO DO PLANEJAMENTO, DESENVOLVIMENTO E GESTÃO, 2017).

(2) Vale explicar que houve alterações entre as fases do programa com o intuito de melhorar seus resultados, como por exemplo, a possibilidade de uso misto (residencial e comercial) e a diminuição do número máximo de unidades por empreendimento.

(3) Também foram considerados outros campos de busca nos locais em que isso foi possível, tais como: assunto e documento completo. 
(4) Em inglês: 1) "evaluation method" e "social housing"; 2) "assessment method" e "social housing"; 3) "analysis method" e "social housing"; 4) "method of evaluation" e "social housing"; 5) "method of assessment" e "social housing"; 6) "method of analysis" e "social housing"; 7) "evaluation protocol" e "social housing"; 8) "quality indicator" e "social housing"; 9) "evaluation methodology" e "social housing"; 10) "qualitative analysis" e "social housing"; 11) "quality perception" e "social housing"; 12) "critical analysis" e "social housing" 13) "ex ante assessment" e "social housing"; 14) "ex ante analysis" e "social housing". Em português: 15) "método de avaliação" e "habitação social"; 16) "método de análise" e "habitação social"; 17) "protocolo de avaliação" e "habitação social"; 18) "indicador de qualidade" e "habitação social"; 19) "metodologia de avaliação" e "habitação social"; 20) "análise qualitativa" e "habitação social"; 21) "percepção de qualidade" e "habitação social"; 22) "análise crítica" e "habitação social"; 23 ) "avaliação ex ante" e "habitação social"; 24) "análise ex ante" e "habitação social". Em espanhol: 25) "método de evaluación" e "vivienda social"; 26) "método de evaluación" e "vivienda protegida"; 27) "método de análisis" e "vivienda social"; 28) "método de análisis" e "vivienda protegida"; 29) "protocolo de evaluación" e "vivienda social"; 30) "protocolo de evaluación" e "vivienda protegida"; 31) "indicador de calidad" e "vivienda social"; 32) "indicador de calidad" e "vivienda protegida"; 33) "metodología de evaluación" e "vivienda social"; 34) "metodología de evaluación" e "vivienda protegida"; 35) "análisis cualitativo" e "vivienda social"; 36) "análisis cualitativo" e "vivienda protegida"; 37) "percepción de la calidad" e "vivienda social"; 38) "percepción de la calidad" e "vivienda protegida"; 39) "análisis critico" e "vivienda social”; 40) "análisis critico" e "vivienda protegida"; 41) "evaluación ex ante" e "vivienda social"; 42) "evaluación ex ante" e "vivienda protegida"; 43) "análisis ex ante" e "vivienda social"; 44) "análisis ex ante" e "vivienda protegida".

(5) Nessa etapa, as revistas pesquisadas foram: 1.) Categoria Architecture: Journal of Building Performance Simulation; Research in Engineering Design - Theory, Applications, and Concurrent Engineering; Design Studies; Structural Design of Tall and Special Buildings; Architectural Science Review; Journal of Architectural Engineering; Geotechnical and Geological Engineering; CoDesign; Tumu Gongcheng Xuebao/China Civil Engineering Journal; Construction Innovation; International Journal of Architectural Heritage; Engineering, Construction and Architectural Management; Facilities; International Journal of Low Carbon Technologies; Architectural Engineering and Design Management; International Journal of Space Structures; Frontiers of Architectural Research; Informes de la Construccion; Journal of Architecture; Structural Engineer; Journal of Asian Architecture and Building Engineering. 2.) Categoria Urban Studies: Journal of Urban Economics; International Journal of Urban and Regional Research; European Urban and Regional Studies; Urban Studies; City; Journal of the American Planning Association; Urban Geography; City and Community; Cities; Environment and Urbanization; Urban Affairs Review; Housing, Theory and Society; Regional Science and Urban Economics; Housing Policy Debate; Urban Ecosystems; Journal of Real Estate Finance and Economics; Journal of Urban Affairs; Urban Education; Journal of Planning Education and Research; Housing Studies; Economic Development Quarterly; Journal of Transport and Land Use; Journal of Urban Technology; Journal of Contemporary Ethnography; Journal of Public Transportation; IATSS Research; Journal of the Urban Planning and Development Division; Urban Design International; European Journal of Transport and Infrastructure Research; Urban Forum; Built Environment; Urban Climate; Journal of Housing and the Built Environment. 3.) Categoria Nature and Landscape Conservation: Conservation Letters; Conservation Biology; Wildlife Monographs; Ecosystem Services; Biological Conservation; Landscape Ecology; Animal Conservation; Landscape and Urban Planning; Sustainability Science; Forest Ecology and Management; Land Use Policy; Journal of Mammalogy; Environmental Conservation; Ecology and Evolution; International Journal of Tourism Research; Ecological Engineering; Journal of Wildlife Management; Biodiversity and Conservation; Endangered Species Research; Habitat International; Oryx; Ibis; Conservation and Society; Journal of Insect Conservation; Restoration Ecology; Applied Vegetation Science; Aquatic Conservation: Marine and Freshwater Ecosystems; Rangeland Ecology and Management; European Journal of Wildlife Research. 4.) Categoria Building and Construction: Cement and Concrete Research; Cement and Concrete Composites; Journal of Structural Engineering; Energy and Buildings; Journal of Composites for Construction; Building and Environment; Tunnelling and Underground Space Technology; International Journal of Refrigeration; Journal of Constructional Steel Research; Indoor Air; Structural Safety; Automation in Construction; Journal of Earthquake Engineering; ACI Structural Journal; Bulletin of Earthquake Engineering; Thin-Walled Structures; Construction and Building Materials; Structural Control and Health Monitoring; Journal of Building Performance Simulation; ACI Materials Journal; Building Research and Information; Journal of Construction Engineering and Management - ASCE; Construction Management and Economics; Journal of Materials in Civil Engineering; Structure and Infrastructure Engineering; Structural Concrete; Journal of Bridge Engineering; Materials and Structures/Materiaux et Constructions; Journal of Building Physics; PCI Journal; Magazine of Concrete Research; Georisk; Jianzhu Jiegou Xuebao/Journal of Building Structures; Structural Design of Tall and Special Buildings; Building Simulation; Journal of Advanced Concrete Technology; Electronic Journal of Information Technology in Construction; Materiales de Construccion.

(6) Por exemplo, no caso do BREEAM (Building Research Establishment Environmental Assessment Method) as categorias de "bem-estar", "transporte", "inovação" e de "uso do solo" estão melhor alinhadas ao interesse da pesquisa do que as restantes. Já no método CASBEE (Comprehensive Assessment Scheme for Building Environmental Efficiency), a categoria sobre "qualidade ambiental da construção" é de maior interesse. 
(7) O número de publicações no gráfico 3 é maior do que o total encontrado na RSL porque em alguns casos foi possível encontrar uma mesma publicação utilizando diferentes palavras-chave de busca. A publicação foi considerada mais de uma vez nos casos em que as palavras estavam em conjuntos distintos.

(8) A partir desse programa, surgiu a possibilidade da Companhia de Desenvolvimento Habitacional e Urbano do Estado de São Paulo (CDHU-SP) exigir a qualificação de seus fornecedores - conforme a Norma Brasileira NBR ISO 9002 - através do Programa Setorial de Qualidade (PSQ) (GALVÃO; ORNSTEIN; ONO, 2013; MELHADO, 2004).

(9) Esse programa é utilizado pela Caixa Econômica Federal desde 2001 como uma das exigências para o financiamento habitacional. Apenas as empresas construtoras que estão qualificadas pelo programa podem receber financiamento.

\section{Referências}

AMORE, C. S., SHIMBO, L. Z., RUFINO, M.B. C. (Org.). Minha Casa e a Cidade? Avaliação do Programa Minha Casa Minha Vida em seis estados Brasileiros. 1ed. Rio de Janeiro, Letra Capital, 2015.

ABNT - ASSOCIAÇÃO BRASILEIRA DE NORMAS TÉCNICAS. NBR 15575: Desempenho de Edificações Habitacionais. Rio de Janeiro: ABNT, 2013.

BONDUKI, N. Política habitacional e inclusão social no Brasil: revisão histórica e novas perspectivas no governo Lula. São Paulo, ARQ.URB, n.1, p. 70-101, 2008. Disponível em: < http://www.usjt.br/arq.urb/numero_01/artigo_05_180908.pdf>. Acesso em: $22 / 03 / 2018$

BONDUKI, N. Do Projeto Moradia ao Programa Minha Casa Minha Vida. TD. Teoria e Debate, v. 82, p. 1, 2009.

CAMPOS, V. F. Controle da Qualidade Total. Rio de Janeiro: Editora Bloch, $3^{\text {a }}$ edição, 1992.

CARDOSO, A. L. (Org.). O programa Minha Casa Minha Vida e seus efeitos territoriais. $1^{\text {a }}$ ed. Rio de Janeiro: Letra Capital, 2013.

CARMONA, M. et al.. Public Places - Urban Spaces: the dimensions of urban design. $1^{\mathrm{a}}$ ed. Architectural Press, 2003.

DINIZ, M. T.; FEDELI, M. T. (Org.). Diretrizes e Instrumentos de Avaliação de Projetos. In: FRANÇA; COSTA (Org.). Do plano ao projeto: novos bairros e habitação social em São Paulo. Política Municipal de Habitação - Prefeitura de São Paulo (volume 2), 2012, p. 179-313.

DRESCH, A.; LACERDA, D. P.; ANTUNES JÚNIOR, J. A. V. Design Science Research: método de pesquisa para avanço da ciência e tecnologia. Porto Alegre: Bookman, 2015.

ELOY, C. M.; COSTA, F.; ROSSETTO, R. Direito à moradia no Brasil: A política de subsídios habitacionais. In: FONSECA, Ana (Org.); FAGNANI, Eduardo (Org.). Políticas sociais, desenvolvimento e cidadania: Educação, seguridade social, pobreza, infraestrutura urbana e transição demográfica. São Paulo: Editora Fundação Perseu Abramo, v. 2, 2013, p. 337-368.

FERREIRA, J. S. W. (Coord.). Produzir casas ou construir cidades? Desafios para um novo Brasil Urbano. $1^{\text {a }}$ ed. São Paulo: Fupam, 2012.

GALVÃO, W. J. F.; ORNSTEIN, S. W.; ONO, R. A avaliação pós-ocupação em empreendimentos habitacionais no Brasil: da reabilitação aos novos edifícios. In: Villa, S. B.; Ornstein, S. W. (Orgs.). Qualidade Ambiental na Habitação: avaliação pósocupação. São Paulo: Oficina de Textos, 2013. p. 37-50.

GANN, D. M.; WHYTE, J. K.. Design quality, its measurement and management in the built environment. Building Research \& Information, v. 31, n. 5, p. 314-317, 2003. ISSN: 1466-4321. https://doi.org/10.1080/0961321032000107546

GANN, D.; SALTER, A.; WHYTE, J. Design quality indicator as a tool for thinking. Building Research \& Information, v.31, n. 5, p. 318-333, 2003. ISSN: 1466-4321. https://doi.org/10.1080/0961321032000107564

GERRITSE, K. Controlling costs and quality in the early phases of the accommodation process. Delft: VSSD, 2008.

GIL, A. C. Como elaborar projetos de pesquisa. 5a ed. São Paulo: Atlas, 2010. 
JONES, J. C. A Method of Systematic Design. In: CROSS, Nigel. Developments in Design Methodology. John Wiley \& Sons, 1984. p. 9-30.

KERNAHAN, D. et al.. User participation in building design and management. Butterworth Architecture, Oxford, 1992.

KOWALTOWSKI, D. C. C. K.; GRANJA, A. D.; MOREIRA D. de C.; SILVA, V. G. da S.; PINA, S. A. M. Métodos e instrumentos de avaliação de projetos destinados à habitação social. In: ORNSTEIN, Sheila Walbe; VILLA, Simone Barbosa (Org.). Qualidade Ambiental na Habitação: avaliação pós-ocupação. São Paulo: Oficina de Textos, 2013. p. 149-184.

KOWALTOWSKI, D. C. C. K. et al.. The Brazilian Housing program "Minha Casa Minha Vida": a systematic literature review. Journal of the Korean Housing Association, v. 26, n. 6, p. 35-42, 2015. https://doi.org/10.6107/JKHA.2015.26.6.035

KRAUSE, C.; BALBIM, R.; LIMA NETO, V. C.. Minha Casa Minha Vida, Nosso Crescimento: Onde Fica a Política Habitacional? Brasília: IPEA, 2013.

KLINK; J.; DENALDI, R. On financialization and state spatial fixes in Brazil. A geographical and historical interpretation of the housing program My House My Life. Habitat International, v. 44, p. 220-226, 2014. ISSN: 0197-3975.

https://doi.org/10.1016/j.habitatint.2014.06.001

LAWSON, B. How designers think: the design process demystified. $3{ }^{\text {a }}$ ed. Oxford: Architectural, 1997.

LOPES, J. M. de A.; SHIMBO, L. Z. Projeto e produção da habitação na região central do estado de São Paulo: condições e contradições do PMCMV. In: AMORE, C. S., SHIMBO, L. Z., RUFINO, M.B. C. (Org.). Minha Casa e a Cidade? Avaliação do Programa Minha Casa Minha Vida em seis estados Brasileiros. 1ed. Rio de Janeiro, Letra Capital, 2015, p. $229-253$.

MACMILLAN, S.. Added value of good design. Building Research \& Information, v. 34, n. 3, p. 257-271, 2006. ISSN: 14664321. https://doi.org/10.1080/09613210600590074

MARICATO, E. É preciso repensar o modelo. Revista Arquitetura e Urbanismo, n. 186, p. 44-49, set. 2009. Disponível em: $<$ http://au17.pini.com.br/arquitetura-urbanismo/186/artigo151670-1.aspx>. Acesso em: 22 mar. 2018.

MARQUES, Eduardo; RODRIGUES, Leandro. O Programa Minha Casa Minha Vida na metrópole paulistana: atendimento habitacional e padrões de segregação. Revista Brasileira de Estudos Urbanos e Regionais, [S.1.], v. 15, n. 2, p. 159 , nov. 2013. ISSN 2317-1529. doi: http://dx.doi.org/10.22296/2317-1529.2013v15n2p159.

MEDRANO, L.; SPINELLI, J. Urban policies and projects for social housing in central areas. The case of the Habitasampa competition (São Paulo, Brazil). Habitat International, v. 42, p. 39-47, 2014. https://doi.org/10.1016/j.habitatint.2013.10.004

MELHADO, S. B. Qualidade e avaliação de desempenho no processo de projeto. In: Seminário Internacional NUTAU, 2004, São Paulo. Artigo técnico. 2004.

MINISTÉRIO DO PLANEJAMENTO, DESENVOLVIMENTO E GESTÃO. $4^{\circ}$ Balanço do PAC 2015-2018. Brasília: Governo Federal, 2017.

MONTANER, Josep Maria. Arquitetura e critica. Barcelona: G. Gili, 2007. 160 p. ISBN 9788425222382 (broch.).

NAGLE, C. B.; RAPPL, K.; MEDRANO, L. S. Métodos de análise de projetos habitacionais: Uma discussão sobre o método I+D+VS. Gestão de Tecnologia de Projetos, São Paulo, v. 8, n. 2, p. 105-115, dec. 2013. ISSN 1981-1543. doi: http://dx.doi.org/10.11606/gtp.v8i2.80952

PEQUENO, R.; ROSA, S. V. Inserção urbana e segregação espacial: análise do Programa Minha Casa Minha Vida em Fortaleza. In: AMORE, C. S., SHIMBO, L. Z., RUFINO, M.B. C. (Org.). Minha Casa e a Cidade? Avaliação do Programa Minha Casa Minha Vida em seis estados Brasileiros. 1ed. Rio de Janeiro, Letra Capital, 2015, p. 131-164.

PEREIRA, Carolina Celete Pinto. Habitação coletiva de interesse social e cidade: análise dos concursos Renova SP (São Paulo, Brasil) e Vallecas 49 (Madri, Espanha). 2014. 174 p. Dissertação (mestrado) - Universidade Estadual de Campinas, Faculdade de Engenharia Civil, Arquitetura e Urbanismo, Campinas, SP. Disponível em:

$<$ http://www.repositorio.unicamp.br/handle/REPOSIP/258065>. Acesso em: 22 mar. 2018. 
PRASAD, S. Clarifying intentions: the design quality indicator. Building Research \& Information, v. 32, n. 6, p. 548-551, 2004. ISSN: 1466-4321. https://doi.org/10.1080/0961321042000312376

PULTAR, Mustafa. A Conceptual Framework for Values in the Built Environment. In: ANNUAL CONFERENCE OF THE INTERNATIONAL ASSOCIATION FOR PEOPLE-ENVIRONMENT STUDIES, 14., Stockholm, 1996. Proceedings ... Stockholm: Royal Institute of Technology, 1996. p. 261-267. Disponível em: < https://iaps.architexturez.net/doc/oai-iaps-id1202bm1030>. Acesso em: 22 mar. 2018.

RAPPL, K. Políticas públicas e habitação de interesse social: similaridades e diferenças entre o caso Brasileiro e o Espanhol. 2015. Dissertação (Mestrado) - Universidade Estadual de Campinas. Disponível em:

$<$ http://repositorio.unicamp.br/jspui/handle/REPOSIP/258070>. Acesso em: 22 mar. 2018.

ROLNIK, R. (Coord.); BISCHOF, R.; KLINTOWITZ, D.; REIS, J. Como produzir moradia bem localizada com recursos do programa minha casa minha vida? Implementando os instrumentos do Estatuto da Cidade. Brasília: Ministério das Cidades, 2010 .

ROLNIK et al. Ferramentas para avaliação da inserção urbana dos empreendimentos do MCMV. São Paulo: FAU-USP, 2014. (Relatório de Pesquisa MCTI/CNPq/MCIDADES Nº 11/2012)

RUFINO, M. B. C. Um olhar sobre a produção do PMCMV a partir de eixos analíticos. In: AMORE, C. S., SHIMBO, L. Z., RUFINO, M.B. C. (Org.). Minha Casa e a Cidade? Avaliação do Programa Minha Casa Minha Vida em seis estados Brasileiros. 1ed. Rio de Janeiro, Letra Capital, 2015, p. 51-70.

SAUNDERS, W. S. (Ed.). Judging Architectural Value. University of Minnesota Press, 2007.

SOUSA, M. J. C. N. C. Aplicação do método SEL a projectos de edifícios da habitação elaborados em Portugal. 1994. Dissertação (Mestrado) - Universidade do Porto, Porto.

THOMSON, D. S. et al.. Managing value and quality in design. Building Research \& Information, v. 31, n. 5, p. 334-345, 2003. ISSN: 1466-4321. https://doi.org/10.1080/0961321032000087981

VALENÇA, M. M.; BONATES, M. F. The trajectory of social housing in Brazil: From the National Housing Bank to the Ministry of the Cities. Habitat International, v. 34, n.2, p. 165-173, abr. 2010. ISSN: 0197-3975.

https://doi.org/10.1016/j.habitatint.2009.08.006

VOORDT, Theo J. M. Van Der; WEGEN, Herman B. R. Van. Arquitetura sob o olhar do usuário: programa de necessidades, projeto e avaliação de edificações. São Paulo: Oficina de Textos, 2013.

VOLKER, L. Deciding about Design Quality: value judgements and decision making in the selection of architects by public clients under European tendering regulations. Leiden: Sidestone Press, 2010.

WHYTE, J. K.; GANN, D. M. Design quality indicators: work in progress. Building Research \& Information, v. 31, n. 5, p. 387398, 2003. ISSN: 1466-4321. https://doi.org/10.1080/0961321032000107537

ZEIN, R. V. O lugar da crítica: ensaios oportunos de arquitetura. Porto Alegre: Faculdades Integradas do Instituto Ritter dos Reis, 2001.

\footnotetext{
${ }^{\text {i }}$ Katrin Rappl

Arquiteta Urbanista. Mestre em Arquitetura, Tecnologia e Cidade pela Universidade Estadual de Campinas. Doutoranda no Programa de Pós-Graduação em Arquitetura e Urbanismo, Universidade de São Paulo. Endereço Postal: Rua do Lago, 876, São Paulo, SP, Brasil, 03178-200.

\section{ii Leandro Silva Medrano}

Arquiteto Urbanista. Professor Livre-docente do Departamento de História da Arquitetura e Estética do Projeto da Faculdade de Arquitetura e Urbanismo da Universidade de São Paulo. Endereço Postal: Rua do Lago, 876, São Paulo, SP, Brasil, 03178-200.
} 